\title{
Hypnotherapy for the Management of Chronic Pain
}

\author{
Gary Elkins ${ }^{1}$, \\ Texas A \& M University College of Medicine and Scott and White Clinic and Hospital, Temple, Texas, \\ USA \\ Mark P. Jensen, and \\ University of Washington School of Medicine, Seattle, Washington, USA \\ David R. Patterson \\ University of Washington School of Medicine, Seattle, Washington, USA
}

\begin{abstract}
This article reviews controlled prospective trials of hypnosis for the treatment of chronic pain. Thirteen studies, excluding studies of headaches, were identified that compared outcomes from hypnosis for the treatment of chronic pain to either baseline data or a control condition. The findings indicate that hypnosis interventions consistently produce significant decreases in pain associated with a variety of chronic-pain problems. Also, hypnosis was generally found to be more effective than nonhypnotic interventions such as attention, physical therapy, and education. Most of the hypnosis interventions for chronic pain include instructions in self-hypnosis. However, there is a lack of standardization of the hypnotic interventions examined in clinical trials, and the number of patients enrolled in the studies has tended to be low and lacking long-term follow-up. Implications of the findings for future clinical research and applications are discussed.
\end{abstract}

Pain that persists for longer than 6 months is referred to as chronic pain (Keefe, 1982). Unrelieved chronic pain can cause considerable suffering, physical limitations, and emotional distress (Turk, 1996). Further, chronic pain is one of the most common reasons for seeking medical care but often persists despite treatment with analgesics and physical modalities. For example, epidemiologic studies indicate that approximately $11 \%$ to $45 \%$ of individuals in the United States experience chronic back pain (LeResche \& Von Korff, 1999), 75\% of patients with advanced cancer suffer persistent pain (Bonica, 1990), and chronic pain is the most common reason for the use of complementary and alternative therapies (Astin, 1998; Eisenberg et al., 1993).

Interest in hypnosis for pain management has increased with recent evidence that hypnosis can reduce pain (and costs) associated with medical procedures (Lang et al., 2000), and there are now an adequate number of controlled studies of hypnosis to draw meaningful conclusions from the literature regarding chronic pain (Jensen \& Patterson, 2006; Montgomery, DuHamel, \& Redd, 2000; Patterson \& Jensen, 2003). Hypnosis in the treatment of chronic pain generally, but not always, involves a hypnotic induction with suggestions for relaxation and comfort. Posthypnotic suggestions may be given for reduced pain that can continue beyond the session or that the patient can quickly and easily create a state of comfort using a cue (i.e., taking a deep breath and exhaling as eye lids close). The focus of hypnosis in the treatment of chronic pain also often involves teaching the patient self-hypnosis or providing tape recordings of hypnosis sessions that can be used to reduce pain on a daily basis outside the sessions. In our

1Address correspondence to Gary Elkins, Department of Psychiatry and Behavioral Sciences (57th Street Annex), Scott and White Clinic and Hospital, 2401 South 31st Street, Temple, TX 76501, USA. E-mail: gelkins@swmail.sw.org. 
experience, some patients experience an immediate reduction in pain severity following hypnosis treatment, whereas others can obtain reduction in pain with repeated practice of selfhypnosis or hypnosis sessions.

The purpose of the present paper is to evaluate the efficacy of hypnosis for the treatment of chronic pain as determined by a review of controlled prospective trials. Studies are reviewed with regard to types of chronic-pain problems treated with hypnosis. This state-of-the-science review includes some recently published clinical trials that have not been included in any previous reviews, as well as a discussion of the implications of the findings for future research and clinical applications.

\section{Controlled Trials of Hypnosis in the Treatment of Chronic-Pain Problems}

Thirteen studies, excluding studies of headaches (note: hypnosis in the treatment of headaches is reviewed elsewhere in this issue) were identified that compared outcomes from hypnosis in the treatment of chronic pain to either baseline data or a control condition. Hypnosis has been applied to a variety of chronic-pain conditions including those from cancer (Elkins, Cheung, Marcus, Palamara, \& Rajab, 2004; Spiegel \& Bloom, 1983), low-back problems (McCauley, Thelen, Frank, Willard, \& Callen, 1983; Spinhoven \& Linssen, 1989), arthritis (Gay, Philippot, \& Luminet, 2002), sickle cell disease (Dinges et al., 1997), temporomandibular conditions (Simon \& Lewis, 2000; Winocur, Gavish, Emodi-Perlman, Halachmi, \& Eli, 2002), fibromyalgia (Haanen et al., 1991), physical disability (Jensen et al., 2005), and mixed etiologies (Appel \& Bleiberg, 2005-2006; Edelson \& Fitzpatrick, 1989; Melzack \& Perry, 1975). These studies are reviewed in regard to research design and outcomes for each chronicpain condition.

\section{Cancer Pain}

Spiegel and Bloom (1983) assigned 54 women with chronic cancer pain from breast carcinoma to either standard care $(n=24)$ or weekly expressive-supportive group therapy for up to 12 months $(n=30)$. The women randomized to the group therapy condition were assigned to groups that either did or did not have self-hypnosis training as a part of their treatment. The hypnosis intervention was directed toward enhancing patient competence and mastery in managing pain and stress related to cancer. Hypnotic training included suggestions to "filter out the hurt" of any sensations by imagining competing sensations in affected areas. Patients were also given instructions for using self-hypnosis outside of the group-therapy sessions. Both treatment groups demonstrated significantly less pain and suffering than the control sample. Hypnosis was not the main focus of the expressive-supportive group-therapy sessions, however, patients who received hypnosis in addition to group therapy reported significantly $(p<.05)$ less increase in pain over time (as cancer progressed) compared to patients who did not receive the hypnosis intervention.

Elkins et al. (2004) conducted a prospective, randomized study of 39 advanced-stage (Stage III or IV) cancer patients with malignant bone disease. Patients were randomized to receive either weekly sessions of supportive attention or a hypnosis intervention. Patients assigned to the hypnosis intervention received at least four weekly sessions in which a hypnotic induction was completed following a standard transcript. The transcript included suggestions for relaxation, comfort, mental imagery for dissociation and pain control, and instruction in selfhypnosis. In addition, patients in the hypnosis intervention were provided with an audiocassette tape recording of a hypnotic induction and instructed in home practice of hypnosis. The hypnosis intervention group demonstrated an overall decrease in pain $(p<.0001)$ for all sessions combined. The mean rating of the effectiveness of self-hypnosis practice outside the sessions was 6.5 on a 0 -to- 10 scale. 


\section{Low-Back Pain}

McCauley et al. (1983) conducted a prospective trial comparing hypnosis and relaxation training for chronic low-back pain. Seventeen outpatients were assigned to either self-hypnosis $(n=9)$ or relaxation $(n=8)$. The baseline was an EMG-assessment session and 1 week later the patients began eight individual weekly sessions. No significant change in any outcome measure was observed during the 1-week baseline period. Patients were assessed 1 week after the completion of treatment and then again 3 months after the treatment ended. Patients in both groups were found to have significant reductions in pain as measured by the McGill Pain Questionnaire and visual analog ratings of pain. Patients given the hypnosis intervention reported significant pre- to posttreatment (percent improvement in the three pain measures were $31 \%, 25 \%$, and $25 \%$, respectively) and pretreatment to 3-month follow-up improvement. However, both the hypnosis intervention and relaxation were effective; neither proved to be superior to the other.

Spinhoven and Linssen (1989) compared training in self-hypnosis to an education program for chronic low-back-pain patients using a crossover study design. Forty-five patients with lowback pain were assigned to receive one of the two treatments first, followed by 2 months of no treatment/follow-up, then the treatment that they had not yet received, followed by another 2month follow-up period. A pain diary was used as a measure of pain intensity, up-time, and use of pain medication. Distress and depression were assessed using the Symptom Checklist-90 (SCL-90). Patients in the hypnosis condition received hypnosis that included a variety of suggestions such as relaxation, imaginative inattention, pain displacement, pain transformation, and future-orientated imagery. Patients were taught to use self-hypnosis and in the fifth session they were given an audiotape to facilitate continued self-hypnosis practice. Patients in the education condition received lectures and facilitated discussion to induce an attitude of self-control of pain. A number of patients dropped out of this study; however, the data that were available from the 24 patients who completed both phases of the study (and therefore received both treatments) showed significant pretreatment to 2-month follow-up improvement on all outcome measures except pain intensity. Further, the post hoc analyses did not reveal any significant differences between the two treatment conditions on any measure. It was concluded that the treatment package was effective in teaching patients with chronic low-back pain to better cope with their pain and to achieve improved adjustment to chronic pain.

\section{Arthritis Pain}

Gay et al. (2002) compared the effectiveness of hypnosis and Jacobson relaxation for the reduction of osteoarthritis pain. Thirty-six patients with osteoarthritis pain were randomly assigned to one of three conditions: hypnosis, relaxation training, and a no-treatment/standardcare control condition. The hypnosis intervention consisted of eight weekly sessions that began with a standard relaxation induction followed by suggestions for positive imagery, as well as a memory from childhood that involved joint mobility. The subjects in the standard-care control condition were administered the outcome measures and were offered treatment after their last follow-up assessment. Patients in the hypnosis treatment showed a substantial and significant decrease in pain intensity after 4 weeks of treatment, which was maintained through 3 months and 6 months of follow-up. In comparison, patients in the no-treatment control condition reported little change in pain during the 6 months of this trial. However, although significant differences between the hypnosis and the standard-care control condition were found midtreatment (4 weeks after treatment started), posttreatment, and at follow-up, the differences between the effects of the hypnosis intervention and the relaxation control on pain reduction were not statistically different. 


\section{Sickle Cell Disease}

Dinges et al. (1997) enrolled 37 children and adults with sickle cell disease (SCD) who reported experiencing episodes of vaso-occlusive pain into a prospective 2-year treatment protocol. A pre- and postexperimental design was used and participants were asked to complete daily diaries during 4 months of baseline and during the 18 months of treatment that involved weekly (for the first 6 months), bimonthly (for the next 6 months), and once every 3 weeks (for the final 6 months) cognitive-behavioral intervention that centered on self-hypnosis training and practice. The hypnosis intervention included suggestions for ideomotor responses (e.g., hands moving together, arm becoming lighter and rising) and encouragement to develop individualized metaphors and self-suggestions to use for pain management. Results indicated the self-hypnosis intervention was associated with a significant reduction in the number of pain days. There were significant baseline versus treatment phase differences observed on: (a) the percentage of days during which both SCD pain (from 20 to 11 days) and non-SCD pain from (19 to 6 days) were reported by patients, (b) percentage of days of non-SCD pain that medication was taken (from 6\% to 1\%), and (c) percentage of "bad sleep nights" on non-SCD pain days (from $8 \%$ to $2 \%$ ). No significant changes were found in the percentage of days of SCD pain that medication was taken or on the percentage of bad sleep nights on SCD pain days, however. The authors concluded that the overall reduction in pain frequency was due to the elimination of less severe episodes of pain.

\section{Temporomandibular Pain}

Temporomandibular disorder can be associated with chronic pain related to dysfunction of the masticatory musculature, the temporomandibular joint, or both. Simon and Lewis (2000) examined the effectiveness of hypnosis on temporomandibular pain disorder in 28 patients. Measures of pain symptoms (pain intensity, duration, and frequency) were assessed on four separate occasions: during wait list, before treatment, after treatment, and at 6-month followup. The hypnosis intervention consisted of education about hypnosis and five sessions that involved an eye-closure induction, relaxation imagery, suggestions for limb catalepsy, metaphors, suggestions for hypnotic analgesia and anesthesia suggestions, and suggestions that muscle tension would serve as a cue for relaxation. Patients were also instructed to practice self-hypnosis daily with audiotaped recordings of the hypnotic treatment. The results indicated a significant decrease in pain frequency $(p<.001)$, pain duration $(p<.001)$, and an increase in daily functioning. Analyses also suggested that the treatment gains were maintained for 6 months after treatment with reduced pain and improved daily functioning.

Winocur et al. (2002) compared "hypnorelaxation" to the use of an occlusal appliance or an education and advice condition for the treatment of temporomandibular pain. The study sample consisted of 40 female patients who were randomly assigned to the three treatment groups: (a) hypnorelaxation $(n=15)$; (b) occlusal appliance $(n=15)$; and (c) education/advice $(n=10)$. The hypnorelaxation intervention included progressive muscle relaxation suggestions and selfhypnosis training for relaxation of facial muscles. Patients in the occlusal appliance condition were provided with a full-coverage, hard acrylic appliance constructed to fit the maxillary arch. Patients assigned to the education and advice condition were provided with recommendations regarding how to manage activities and diet in order to better manage pain. Pain intensity (current and worst) was assessed before and after treatment using visual analog ratings. Both active treatment modes (hypnorelaxation and occlusal appliance) were more effective than education/advice in alleviating sensitivity to palpation. However, only patients in the hypnosis condition (not the occlusal appliance condition) reported significantly greater decreases in pain intensity: $57 \%$ reduction for current pain intensity and 51\% reduction for worst pain intensity compared to patients in the education/advice condition. 


\section{Fibromyalgia}

In a controlled study, Haanen et al. (1991) randomly assigned 40 patients with fibromyalgia to groups that received either eight 1-hour sessions of hypnotherapy with a self-hypnosis homepractice tape over a 3-month period, or physical therapy (that included 12 to 24 hours of massage and muscle relaxation training) for 3 months. Outcome was assessed pre- and posttreatment and at 3-month follow-up. The hypnosis intervention included an arm-levitation induction and suggestions for ego strengthening, relaxation, improved sleep, and "control of muscle pain." Compared with patients in the physical therapy group, the patients who received hypnosis showed significantly better outcomes on measures of muscle pain, fatigue, sleep disturbance, distress, and patient overall assessment of outcome. These differences were maintained at the 3-month follow-up assessment and the average percent decrease in pain among patients who received hypnosis $(35 \%)$ was clinically significant, whereas the percent decrease in the patients who received physical therapy was marginal (2\%).

\section{Disability-Related Pain}

Jensen et al. (2005) examined the effects of 10 sessions of standardized (script-driven) hypnotic analgesia treatment on pain intensity, pain unpleasantness, depression, and perceived control over pain in 33 patients with chronic pain secondary to a disability. Outcome measures were assessed before and after a baseline period, as well as after treatment and at 3-month followup. The hypnosis intervention consisted of a hypnotic induction followed by five specific suggestions for alteration of pain: diminution of pain, relaxation, imagined analgesia, decreased pain unpleasantness, and replacement of pain with other nonpainful sensations. Also, posthypnotic suggestions were given for daily practice of hypnosis but the patients in the study were not given any practice tapes prior to the 3-month follow-up assessment. Analyses indicated significant pre- to posttreatment improvement in pain intensity, pain unpleasantness, and perceived control over pain (but not depressive symptoms) over and above change that occurred during the baseline period. Improvement was also maintained at the 3-month followup. Hypnotizability and concentration of treatment (e.g., daily vs. weekly) were not significantly associated with treatment outcome. However, cognitive expectancies assessed after the first session showed a moderate association with pain reduction.

\section{Mixed Chronic-Pain Problems}

Melzack and Perry (1975) examined the effects of hypnosis and neurofeedback in 24 patients who had a variety of chronic-pain problems. Baseline data was collected during two notreatment (baseline) sessions, and patients were then randomly assigned to one of three treatment conditions: four sessions of hypnosis alone, eight sessions of neurofeedback training alone, or both hypnosis and neurofeedback training. The hypnosis treatment consisted of a taped hypnotic induction with suggestions for relaxation, ego strengthening, a feeling of greater tranquility, and of being able to overcome things that are ordinarily upsetting and worrying. No direct suggestions for pain control were included in the hypnosis treatment. The McGill Pain Questionnaire was administered before and after each of the baseline, training, and two posttraining practice sessions. There was a reduction in pain observed during the hypnosis training (range, 21\%-32\% improvement; median improvement $=23 \%$ ), however, none of the observed changes in either the neurofeedback or hypnosis conditions were statistically significant in comparison to the baseline phase.

Edelson and Fitzpatrick (1989) evaluated hypnosis and cognitive-behavior therapy for treatment of chronic pain. Twenty-seven patients with various chronic-pain problems (back pain being the most frequent) were randomly assigned to: cognitive-behavioral therapy (CBT) alone, CBT plus hypnosis treatment, or an attention control (supportive, nondirective discussions). The hypnosis and CBT treatments were identical with the exception of a hypnotic induction. It is noteworthy, however, that the CBT intervention used in this study included 
some what might be considered "hypnotic components." Specifically, the CBT intervention encouraged the participants to: (1) avoid using the "pain" label to describe their sensations; (b) reinterpret pain sensations as "numbness" through the use of imagery (this component, in particular, might be considered as a hypnosis intervention); and (c) monitor and restructure negative self-talk. The results indicated decreases in pain intensity for both the hypnosis intervention and the CBT treatment that were sustained at 1-month follow-up. However, only the CBT treatment resulted in significantly lower pain rating scores in comparison to the attention control condition. In this study, adding a hypnotic induction appeared to have little positive effect. In fact, in this study the CBT treatment minus the induction had a greater effect on pain behaviors. Given the "hypnotic characteristics" of some aspects of the CBT treatment used in this study, this finding is somewhat puzzling. However, this does suggest the possibility that a hypnotic induction may detract from some forms of CBT for chronic pain.

Appel and Bleiberg (2005-2006) investigated the association between hypnotizability and hypnosis for treatment of chronic pain. Twenty-seven patients with a variety of chronic-pain problems (15 lumbar pain, 7 rheumatological pain, 3 cervical pain, 1 peripheral neuropathy, 1 gynecological-related pain) received hypnosis treatment sessions directed at "teaching selfregulation of the affective and sensory components of pain." The word hypnosis was not mentioned during the intervention, which included relaxation training, autogenic statements, guided imagery for pain alteration and health and healing, and individualization to use images "in a way that is best for him or her." The results indicated a significant reduction in pain ratings pre- and posttreatment. Measures of relaxation and suffering were not related to hypnotizability. However, changes in pain ratings were significantly correlated with hypnotizability $(r=.55, p<.001)$ as measured by the Stanford Clinical Hypnotic Scale.

\section{Discussion}

This review identified 13 published controlled articles that evaluated the efficacy of hypnosis for chronic pain. With the exception of two articles (Appel \& Bleiberg, 2005-2006; Melzack \& Perry, 1975), the studies reviewed included a control condition for comparison. In each of the studies, the hypnosis intervention was demonstrated to be significantly more effective than a no-treatment condition in reducing pain in chronic-pain patients. Moreover, the efficacy of hypnosis in reducing pain was consistently confirmed for a wide variety of different chronicpain conditions (e.g., cancer, low-back pain, arthritis pain, sickle cell disease, temporomandibular pain, disability-related pain).

However, there have been a relatively small number of studies conducted for each of the different chronic-pain conditions (in some cases only one study). Although it is encouraging that 13 controlled studies have reported on the use of hypnosis with chronic pain, there are a number of basic research design weaknesses that tend to run throughout most of these reports. The number of patients enrolled in the studies tends to be low, bringing up issues of power to detect group differences. Control conditions used usually have lacked credible controls for placebo and/or expectation. Multiple measures of outcomes are seldom employed as are follow-up assessment of sufficient duration (i.e., long-term follow-up). Thus, although the findings provide support for the general applicability of hypnosis in the treatment of chronic pain, considerably more research will be needed to fully determine the effects of hypnosis for different chronic-pain conditions (e.g., neuropathic, sickle cell disease, arthritis, etc.).

Studies of hypnosis in the treatment of chronic pain have often included instructing patients in self-hypnosis as a way of coping with pain and gaining greater self-control over pain (e.g., Dinges et al., 1997; Elkins et al., 2004; Gay et al., 2002; Haanen et al., 1991; Jensen et al., 2005; McCauley et al., 1983; Simon \& Lewis, 2000; Spiegel \& Bloom, 1983; Spinhoven \& Linssen, 1989; Winocur et al., 2002). This usually includes providing patients with tape 
recordings of hypnosis sessions and instructions in home practice of self-hypnosis. However, research has yet to determine the importance of and the best ways to provide instruction in selfhypnosis practice. For example, it is unknown whether standard tapes are as effective as individualized recordings. Also, the necessary frequency of practice has not been determined or even if home practice is as effective as "live" sessions. Our clinical experience suggests that patients who are more actively involved in self-hypnosis practice benefit more and may have more long-lasting gains (see Elkins et al., 2004; Jensen \& Barber, 2000). In clinical practice, we recommend to patients that they practice at least once a day. To facilitate this, we provide them with tape recordings of the sessions. We also give them instructions for practicing selfhypnosis without the use of a recording. Some patients choose to practice by listening to a tape, and some choose to practice self-hypnosis without a tape; many do both.

Chronic pain is a complex phenomenon that may be affected by emotional, cognitive, behavioral, and physiological responses and a multimodal treatment approach may be important for some chronic-pain patients. However, there have been few studies that have evaluated the effect of hypnosis as an adjunct to other treatment modalities for chronic pain, including, for example, treatment programs designed to increase activity and to reduce the negative effects of pain on function (Patterson \& Jensen, 2003). One study compared CBT to CBT combined with a hypnotic induction. In that study (Edelson \& Fitzpatrick, 1989), only the CBT treatment alone resulted in significantly lower pain-rating scores in comparison to an attention-control condition. This finding is somewhat puzzling, because some aspects of the CBT treatment used in this study appeared to be very similar to a hypnotic intervention (i.e., the CBT intervention included instructions to reinterpret pain sensations as "numbness" through the use of imagery). However, this study suggests the possibility that the addition of a hypnosis induction may have detracted from an intervention focused on altering maladaptive cognitions. Further research is needed to determine the best methods of integrating hypnosis with $\mathrm{CBT}$ and other multimodal interventions for chronic-pain management.

The present review also reveals that there is a lack of standardization in hypnotic induction and interventions. There is a need to more clearly identify the components of a hypnotic intervention to better allow comparison across studies and to differentiate hypnosis from other "hypnotic-like" interventions such as relaxation training. For example, in the present review, treatments such as progressive muscle relaxation and mental imagery appeared to be approximately as effective as interventions that were labeled as "hypnosis." It may be that these treatments are similar in regard to mechanism of action and effect. Research is needed to determine the efficacy of hypnosis and specific hypnotic suggestions and interventions. Jensen and Patterson (2006) proposed a basic chronic-pain hypnotic-analgesia intervention that consists of the following: (a) a standard hypnotic induction that includes a focus of attention and relaxation; (b) suggestions for alteration in subjective experience of pain; (c) hypnotic suggestion lasting at least 20 minutes; (d) four to seven sessions indicating "brief hypnosis treatment" and eight or more sessions to indicate "hypnosis treatment;" and (e) instruction in daily home practice of self-hypnosis. Greater standardization in hypnosis research protocols for chronic pain would allow for greater specificity of treatment and clearer identification of innovations in the development of particularly effective hypnotic interventions.

The current review indicates that hypnotic interventions for chronic pain results in significant reductions in perceived pain that, in some cases, may be maintained for several months. Further, in a few studies, hypnotic treatment was found to be more effective, on average, than some other treatments, such as physical therapy or education, for some types of chronic pain. These findings are encouraging for an initial wave of studies, but a more sophisticated body of research including larger sample sizes and more rigorous controls would be far more convincing. Further, most studies have focused on how hypnotic suggestion may be used to achieve analgesic effect, but hypnosis may also have other benefits for chronic-pain patients 
such as reduced anxiety, improved sleep, and enhanced quality of life (Jensen, McArthur, et al., 2006). These targets for hypnosis intervention with chronic-pain patients warrant further investigation. Research to date has been very promising and continued research is needed to fully evaluate the effects and mechanisms of hypnosis interventions for chronic pain in randomized trials and clinical practice.

\section{References}

Appel PR, Bleiberg J. Pain reduction is related to hypnotizability but not to relaxation or to reduction of suffering: A preliminary investigation. American Journal of Clinical Hypnosis 2005-2006;48:153161. [PubMed: 16482842]

Astin JA. Why patients use alternative medicine: Results of a national study. Journal of the American Medical Association 1998;279:1548-1553. [PubMed: 9605899]

Bonica JJ. Evolution and current status of pain programs. Journal of Pain Symptom Management 1990;5:368-374.

Dinges DF, Whitehouse WG, Orne EC, Bloom PB, Carlin MM, Bauer NK, et al. Self-hypnosis training as an adjunctive treatment in the management of pain associated with sickle cell disease. International Journal of Clinical and Experimental Hypnosis 1997;45:417-432. [PubMed: 9308268]

Edelson J, Fitzpatrick JL. A comparison of cognitive-behavioral and hypnotic treatments of chronic pain. Journal of Clinical Psychology 1989;45:316-323. [PubMed: 2723088]

Eisenberg DM, Kessler RC, Foster C, Norlock FE, Calkins DR, Delbanco TL. Unconventional medicine in the United States. Prevalence, costs, and patterns of use. New England Journal of Medicine 1993;328:246-252. [PubMed: 8418405]

Elkins GR, Cheung A, Marcus J, Palamara L, Rajab H. Hypnosis to reduce pain in cancer survivors with advanced disease: A prospective study. Journal of Cancer Integrative Medicine 2004;2:167-172.

Gay M, Philippot P, Luminet O. Differential effectiveness of psychological interventions for reducing osteoarthritis pain: A comparison of Erikson hypnosis and Jacobson relaxation. European Journal of Pain 2002;6:1-16. [PubMed: 11888223]

Haanen HC, Hoenderdos HT, van Romunde LK, Hop WC, Mallee C, Terwiel JP, et al. Controlled trial of hypnotherapy in the treatment of refractory fibromyalgia. Journal of Rheumatology 1991;18:7275. [PubMed: 2023202]

Jensen MP, Barber J. Hypnotic analgesia of spinal cord injury pain. Australian Journal of Clinical and Experimental Hypnosis 2000;28:150-168.

Jensen MP, Hanley MA, Engel JM, Romano JM, Barber JB, Cardenas DD, et al. Hypnotic analgesia for chronic pain in persons with disabilities: A case series. International Journal of Clinical and Experimental Hypnosis 2005;53:198-228. [PubMed: 16025734]

Jensen MP, McArthur KD, Barber JB, Hanley MA, Engel JM, Romano JM, et al. Satisfaction with, and the beneficial side effects of, hypnosis analgesia. International Journal of Clinical and Experimental Hypnosis 2006;54:432-447. [PubMed: 16950685]

Jensen MP, Patterson DR. Hypnotic treatment of chronic pain. Journal of Behavioral Medicine 2006;29:95-124. [PubMed: 16404678]

Keefe FJ. Behavioral assessment and treatment of chronic pain: Current status and future directions. Journal of Consulting and Clinical Psychology 1982;50:896-911. [PubMed: 7174979]

Lang EV, Benotsch EG, Fick LJ, Lutgendorf S, Berbaum ML, Berbaum KS, et al. Adjunctive nonpharmacological analgesia for invasive medical procedures: A randomised trial. Lancet 2000;355:1486-1490. [PubMed: 10801169]

LeResche, L.; Von Korff, M. Epidemiology of chronic pain. In: Block, AR.; Kemer, EF.; Fernandez, E., editors. Handbook of pain syndromes: Biopsychosocial perspectives. Mahwah, NJ: Lawrence Erlbaum; 1999. p. 3-22.

McCauley JD, Thelen MH, Frank RG, Willard RR, Callen KE. Hypnosis compared to relaxation in the outpatient management of chronic low back pain. Archives of Physical Medicine and Rehabilitation 1983;64:548-552. [PubMed: 6227304]

Melzack R, Perry C. Self-regulation of pain: The use of alpha-feedback and hypnotic training for the control of chronic pain. Experimental Neurology 1975;46:452-469. [PubMed: 1112286] 
Montgomery GH, DuHamel KN, Redd WH. A meta-analysis of hypnotically induced analgesia: How effective is hypnosis? International Journal of Clinical and Experimental Hypnosis 2000;48:138153. [PubMed: 10769981]

Patterson DR, Jensen MP. Hypnosis and clinical pain. Psychological Bulletin 2003;129:495-521. [PubMed: 12848218]

Simon EP, Lewis DM. Medical hypnosis for temporomandibular disorders: Treatment efficacy and medical utilization outcome. Oral Surgery, Oral Medicine, Oral Pathology, Oral Radiology, and Endodontics 2000;90:54-63.

Spiegel D, Bloom JR. Group therapy and hypnosis reduce metastatic breast carcinoma pain. Psychosomatic Medicine 1983;45:333-339. [PubMed: 6622622]

Spinhoven P, Linssen AC. Education and self-hypnosis in the management of low back pain: A component analysis. British Journal of Clinical Psychology 1989;28:145-153. [PubMed: 2525939]

Turk, DC. Biopsychosocial perspective on chronic pain. In: Gatchel, RJ.; Turk, DC., editors. Psychological approaches to pain management: A practitioner's handbook. New York: Guilford; 1996. p. 3-32.

Winocur E, Gavish A, Emodi-Perlman A, Halachmi M, Eli I. Hypnorelaxation as treatment for myofascial pain disorder: A comparative study. Oral Surgery, Oral Medicine, Oral Pathology 2002;93:425-434. 